\title{
DGOR-Jahrestagung 1985
}

Die Deutsche Gesellschaft für Operations Research veranstaltet ihre nächste Jahrestagung

\section{vom 11.-13. September 1985 in Hamburg}

Die Tagung soll über den Stand der Unternehmensforschung in Theorie und Praxis informieren, ein Forum für den Austausch von Erfahrungen sein und Anwendern wie Grundlagenforschern neue Anregungen bieten.

Im wissenschaftlichen Tagungsprogramm sind Übersichtsvorträge und Spezialreferate insbesondere auf folgenden Gebieten vorgesehen:

- Operations Research im Banken- und Versicherungswesen: Investitions- und Finanzplanung, Banken-Controlling, Kalkulation von Versicherungstarifen

- Operations Research in Industrie und Handel: Produktionsplanung, Aktivitätsanalyse, Lagerhaltung, Standortplanung (Logistik), Instandhaltungsplanung, Marketing

- Operations Research in der privatwirtschaftlichen und öffentlichen Verwaltung: Personalplanung, Rechnungswesen, Gesundheitswesen, Strategische Planung, Umweltschutz, Energieplanung

- Informationstechnologie: OR im Personal-Computing, Expertensysteme (Decision Support Systems), Rechnerverbund, OR-Software

- Datenanalyse, Prognoseverfahren, Stochastische Entscheidungsprozesse

- Entscheidungstheorie, Dynamische Optimierung und Kontrolltheorie

- Lineare und Nichtlineare Optimierung, Kombinatorische Optimierung, Fuzzy Set-Theorie, Graphentheorie und Projektmanagement.

Auf Anwendungsberichte aus der Praxis wird besonders Wert gelegt. - Konferenzsprachen sind Deutsch und Englisch.

Es ist vorgesehen, spätere und ausführlichere Darstellungen akzeptierter Beiträge in einem Sammelband der Konferenzergebnisse im Springer-Verlag, Berlin, Heidelberg, New York, Tokyo, zu veröffentlichen.

Programmausschuß: Vorsitzender: Prof. Dr. L. Streitferdt

Universität Hamburg, FB 3 - Wirtschaftswissenschaften

Von-Melle-Park 5, D-2000 Hamburg 13, Telefon: (040) 41 23-55 80/1

Mitglieder: Prof. Dr. H. Hauptmann, Hochschule der Bundeswehr Hamburg

Dipl.-Kfm. A. W. Marusev, Gesellschaft für Automatische Datenverarbeitung e.G.

Prof. Dr. D. Ohse, Universität Frankfurt

Prof. Dr. U. Pape, Technische Universität Berlin

Örtliche Tagungsorganisation: $\quad$ Prof. Dr. W. Domschke und Prof. Dr. W. Junginger

Institut für Unternehmensforschung und Informatik

Holstenhofweg 85, D-2000 Hamburg 70, Telefon: (040) 65 41-27 78/28 57

DEUTSCHE GESELLSCHAFT FUR OPERATIONS RESEARCH (DGOR)

gez. K.P. Schuster (Vorsitzender) Geschäftsstelle: Lärchenweg 9, D-6901 Wilhelmsfeld, Telefon: (06220) 1223 